\title{
A MONGE-AMPÈRE NORM FOR DELTA-PLURISUBHARMONIC FUNCTIONS
}

\author{
URBAN CEGRELL* and JONAS WIKLUND
}

\begin{abstract}
We consider differences of plurisubharmonic functions in the energy class $\mathscr{F}$ as a linear space, and equip this space with a norm, depending on the generalized complex Monge-Ampère operator, turning the linear space into a Banach space $\delta \mathscr{F}$. Fundamental topological questions for this space is studied, and we prove that $\delta \mathscr{F}$ is not separable. Moreover we investigate the dual space. The study is concluded with comparison between $\delta \mathscr{F}$ and the space of delta-plurisubharmonic functions, with norm depending on the total variation of the Laplace mass, studied by the first author in an earlier paper [7].
\end{abstract}

\section{Introduction and notations}

Convex-, subharmonic-, and plurisubharmonic functions are all convex cones in some larger linear space. Given any such cone, $K$ say, we can investigate the space of differences from this cone $\delta K$. Such studies are often motivated by algebraic completion of the cone, and differences of convex functions were considered by F. Riesz in as early as 1911 .

$\delta$-convex functions, or $d . c$. functions as they sometimes are denoted, were studied by Kiselman [15], and Cegrell [8], and have been given attention in many areas ranging from nonsmooth optimization to super-reflexive Banach spaces [13].

$\delta$-subharmonic where first given a systematic treatise in [3]. $\delta$-plurisubharmonic functions were studied by Cegrell [7], and Kiselman [15], where the topology was defined by neighbourhood basis of the form $(U \cap \mathscr{P} \mathscr{S} \mathscr{H})-(U \cap$ $\mathscr{P} \mathscr{S} \mathscr{H}), U$ a neighbourhood of the origin in $L_{l o c}^{1}$.

In this paper we study a subset of $\delta$-plurisubharmonic functions. Let $\Omega$ be a hyperconvex domain in $C^{n}$, then $\mathscr{F}(\Omega)$ is a convex cones in the linear space $L_{l o c}^{1}(\Omega)$. Let $\delta \mathscr{F}(\Omega)$ denote the set of functions $u \in L_{l o c}^{1}(\Omega)$ that can be written as $u=u_{1}-u_{2}$, where $u_{i} \in \mathscr{F}(\Omega)$.

\footnotetext{
* Partially supported by the Swedish Research Council, contract number 621-2002-5308.

Received September 9, 2004.
} 
We will define a norm, depending on the Monge-Ampère operator, for functions in this class and discuss some of the topological questions that this norm raises.

For convenience we will denote the class of negative plurisubharmonic functions on a domain $\Omega$ by $\mathscr{P} \mathscr{S} \mathscr{H}^{-}(\Omega)$, and as in [9] we will denote the class of bounded plurisubharmonic functions with boundary value zero and finite total Monge-Ampère mass by $\mathscr{E}_{0}(\Omega)$.

For the notation of the so called energy class $\mathscr{F}(\Omega)$ on a hyperconvex domain $\Omega$ we refer to the paper [10]. As for now we remind the reader that the generalized complex Monge-Ampère operator is well defined in $\mathscr{F}(\Omega)$, and functions from $\mathscr{F}(\Omega)$ has finite total Monge-Ampère mass, but that the so called "comparison principle" do not hold in general, even if it is true that if $u \geq v$ on $\Omega$ then $\int_{\Omega}\left(d d^{c} u\right)^{n} \leq \int_{\Omega}\left(d d^{c} v\right)^{n}$.

In almost all results in this paper the domain $\Omega$ does not matter much, except for the results in Section 5, and therefore we will often suppress the reference to $\Omega$ from the notation.

This paper is an expanded version of a manuscript that can be found in the second author's doctoral thesis [19]. The authors would like to thank Alexander Rashkovskii and Yang Xing for valuable comments and suggestions.

\section{Definition of the norm}

Definition 2.1. Let $\Omega$ be a hyperconvex set in $C^{n}$. Assume that $u \in \delta \mathscr{F}(\Omega)$, then we define the norm of $u$ to be:

$$
\|u\|=\inf _{\substack{u_{1}-u_{2}=u \\ u_{1}, u_{2} \in \mathscr{F}}}\left[\left(\int_{\Omega}\left(d d^{c}\left(u_{1}+u_{2}\right)\right)^{n}\right)^{\frac{1}{n}}\right] .
$$

Note that for functions $u \in \mathscr{F}$ we have $\|u\|^{n}=\int\left(d d^{c} u\right)^{n}$. To see this choose $u_{2}=0$ in the infimum of the definition and hence $\|u\|^{n} \leq \int\left(d d^{c} u\right)^{n}$. For an inequality in the other direction let $u_{1}, u_{2} \in \mathscr{F}$ be any representation of $u=u_{1}-u_{2}$. Since $u_{2} \leq 0$ we have $u \geq u_{1}-u_{2}+2 u_{2}=u_{1}+u_{2}$, and thus $\int\left(d d^{c} u\right)^{n} \leq \int\left(d d^{c}\left(u_{1}+u_{2}\right)\right)^{n}$ thus $\int\left(d d^{c} u\right)^{n} \leq\|u\|^{n}$.

The following Lemma will be used repeatedly.

Lemma 2.2. Suppose $u, v \in \mathscr{F}, h \in \mathscr{E}_{0}$, and that $p, q$ are positive natural numbers such that $p+q=n$. Then

$$
\int-h\left(d d^{c} u\right)^{p} \wedge\left(d d^{c} v\right)^{q} \leq\left(\int-h\left(d d^{c} u\right)^{n}\right)^{\frac{p}{n}}\left(\int-h\left(d d^{c} v\right)^{n}\right)^{\frac{q}{n}}
$$

Proof. Cf. [10]. 
The following inequality is very useful when working with the MongeAmpère operator, and will be essential for our work.

THeOREM 2.3 (Błocki's inequality, [4]). Let $\Omega$ be an open subset of $\mathrm{C}^{n}$, and let $h, u, v_{1}, \ldots, v_{2} \in \mathscr{P} \mathscr{S} \mathscr{H} \cap \mathscr{C}(\Omega)$. Furthermore, suppose $u \leq h$, and $u=h$ close to $\partial \Omega$, and that $-1 \leq v_{j} \leq 0$ for $1 \leq j \leq n$. Then

$$
\int_{\Omega}(h-u)^{n} d d^{c} v_{1} \wedge \cdots \wedge d d^{c} v_{n} \leq n ! \int_{\Omega}\left(-v_{n}\right)\left(d d^{c} u\right)^{n} .
$$

Lemma 2.4. If $\lambda \in \mathrm{R}$ then $\|\lambda u\|=|\lambda|\|u\|$.

Proof. Let $\lambda \geq 0$. From the definition, we have

$$
\begin{aligned}
\|u\|^{n} & =\inf _{u_{1}-u_{2}=u} \int_{\Omega}\left(d d^{c}\left(u_{1}+u_{2}\right)\right)^{n} \\
& =\inf _{u_{1}-u_{2}=u} \int_{\Omega}\left(d d^{c}\left(\frac{\lambda}{\lambda}\left(u_{1}+u_{2}\right)\right)\right)^{n} \\
& =\inf _{u_{1}-u_{2}=u} \int_{\Omega} \lambda^{-n}\left(d d^{c}\left(\lambda u_{1}+\lambda u_{2}\right)\right)^{n} \\
& =\lambda^{-n} \inf _{\tilde{u}_{1}-\tilde{u}_{2}=\lambda u} \int_{\Omega}\left(d d^{c}\left(u_{1}+u_{2}\right)\right)^{n}=\lambda^{-n}\|\lambda u\|^{n} .
\end{aligned}
$$

Hence $\lambda\|u\|=\|\lambda u\|$.

If $\lambda<0$ we have $\lambda u=-\lambda(-u)$, and the same line of reasoning as above applies.

Lemma 2.5. Suppose $\Omega$ is a hyperconvex domain in $\mathrm{C}^{n}$ and that $u, v \in$ $\mathscr{F}(\Omega)$, then

$$
\int_{\Omega}\left(d d^{c}(u+v)\right)^{n} \leq\left[\left(\int_{\Omega}\left(d d^{c} u\right)^{n}\right)^{\frac{1}{n}}+\left(\int_{\Omega}\left(d d^{c} v\right)^{n}\right)^{\frac{1}{n}}\right]^{n}
$$

Proof. Take $h \in \mathscr{E}_{0}$ and let us consider the left hand side in the inequality above.

$$
\begin{aligned}
\int_{\Omega}-h\left(d d^{c}(u+v)\right)^{n} & =\sum_{j=0}^{n}\left(\begin{array}{c}
n \\
j
\end{array}\right) \int_{\Omega}-h\left(d d^{c} u\right)^{n-j} \wedge\left(d d^{c} v\right)^{j} \\
& \leq \sum_{j=0}^{n}\left(\begin{array}{c}
n \\
j
\end{array}\right)\left(\int_{\Omega}-h\left(d d^{c} u\right)^{n}\right)^{\frac{n-j}{n}}\left(\int_{\Omega}-h\left(d d^{c} v\right)^{n}\right)^{\frac{j}{n}} \\
& =\left[\left(\int_{\Omega}-h\left(d d^{c} u\right)^{n}\right)^{\frac{1}{n}}+\left(\int_{\Omega}-h\left(d d^{c} v\right)^{n}\right)^{\frac{1}{n}}\right]^{n}
\end{aligned}
$$


where the inequality comes from the "Hölder-inequality" in Lemma 2.2. Fix $w \in \Omega$, and take $h=\max \left(k \cdot g_{\Omega},-1\right)$, where $g_{\Omega}(z, w)$ is the pluricomplex Green function with pole at $w$, then $h \in \mathscr{E}_{0}$ and $h \searrow-1$ on $\Omega$ and the Lemma follows.

Now we are in a position to prove the triangle-inequality for $\delta \mathscr{F}$.

Corollary 2.6. Suppose $\Omega$ is a hyperconvex domain in $\mathrm{C}^{n}$ and that $u, v \in$ $\delta \mathscr{F}(\Omega)$, then

$$
\|u+v\| \leq\|u\|+\|v\|
$$

Proof. Take $\epsilon>0$, then there is $u_{i}, v_{i} \in \mathscr{F}$ such that

$$
\left(\int_{\Omega}\left(d d^{c}\left(u_{1}+u_{2}\right)\right)^{n}\right)^{1 / n}<\|u\|+\epsilon
$$

and

$$
\left(\int_{\Omega}\left(d d^{c}\left(v_{1}+v_{2}\right)\right)^{n}\right)^{1 / n}<\|v\|+\epsilon .
$$

According to Lemma 2.5 we have

$$
\begin{aligned}
\|u\|+\|v\|-2 \epsilon & >\left(\int_{\Omega}\left(d d^{c}\left(u_{1}+u_{2}\right)\right)^{n}\right)^{1 / n}+\left(\int_{\Omega}\left(d d^{c}\left(v_{1}+v_{2}\right)\right)^{n}\right)^{1 / n} \\
& \geq\left[\int_{\Omega}\left(d d^{c}\left(u_{1}+u_{2}+v_{1}+v_{2}\right)\right)^{n}\right]^{1 / n},
\end{aligned}
$$

and furthermore, since $u_{1}+v_{1}-\left(u_{2}+v_{2}\right)=u-v, u_{1}+v_{1}$ and $u_{2}+v_{2}$ are two of the functions in the set we take infimum over we have

$$
\left[\int_{\Omega}\left(d d^{c}\left(u_{1}+u_{2}+v_{1}+v_{2}\right)\right)^{n}\right]^{1 / n} \geq\|u+v\| .
$$

Hence $\|u+v\| \leq\|u\|+\|v\|$.

Lemma 2.7. If $\|u\|=0$, then $u=0$.

Proof. Take $\epsilon>0$. Since

$$
\|u\|=\inf _{\substack{u_{1}-u_{2}=u \\ u_{1}, u_{2} \in \mathscr{F}}}\left[\left(\int_{\Omega}\left(d d^{c}\left(u_{1}+u_{2}\right)\right)^{n}\right)^{\frac{1}{n}}\right] .
$$

there is $\tilde{u}_{i} \in \mathscr{F}$ such that $\int_{\Omega}\left(d d^{c}\left(\tilde{u}_{1}+\tilde{u}_{2}\right)\right)^{n}<\epsilon$. 
Take a sequence $\left\{v_{j}\right\} \subset \mathscr{E}_{0} \cap \mathscr{C}(\bar{\Omega})$, such that $v_{j} \searrow \tilde{u}_{1}+\tilde{u}_{2}$ as $j \rightarrow \infty$. Let $t>0$ and define $h_{t}=\max \left\{v_{j},-t\right\}$. According to Błocki's inequality (Theorem 2.3) we have

$$
n ! \epsilon>n ! \int_{\Omega}\left(d d^{c} v_{j}\right)^{n}>\int_{\Omega}\left(h_{t}-v_{j}\right)^{n} d V,
$$

hence

$$
n ! \epsilon>\left\|h_{t}-v_{j}\right\|_{L^{n}} \operatorname{vol}(\Omega)
$$

Letting $t \searrow 0$ we get

$$
\frac{n ! \epsilon}{\operatorname{vol}(\Omega)}>\left\|v_{j}\right\|_{L^{n}}
$$

independent of $j$. Thus $\left\|u_{1}+u_{2}\right\|_{L^{n}}<C \epsilon$, and letting $\epsilon \rightarrow 0$ we get $\|u\|_{L^{n}}=$ 0 , so $u=0$, except for a set of measure zero, but since $u \in \delta \mathscr{F}$ we have $u \equiv 0$.

A remark on other energy classes

Since other type of energy-classes, for instance $\mathscr{E}_{p}(\Omega)$ also are convex cones we can form the linear spaces $\delta \mathscr{E}_{p}$. It is natural to try to generalize our norm to a norm for these spaces. Consider a hyperconvex domain $\Omega \subset \mathrm{C}^{2}$, and the energy class $\mathscr{E}_{1}(\Omega)$. Since $\int_{\Omega}\left(d d^{c} u\right)^{2}$ is not finite in general we have to replace it with $\int_{\Omega}-u\left(d d^{c} u\right)^{2}$. Thus the natural generalization of the norm would be to take $u \in \delta \mathscr{E}_{1}$, and set

$$
q(u)=\inf _{\substack{u_{1}-u_{2}=u \\ u_{1}, u_{2} \in \mathscr{E}_{1}}}\left\{\left(\int_{\Omega}-\left(u_{1}+u_{2}\right)\left(d d^{c}\left(u_{1}+u_{2}\right)\right)^{2}\right)^{\frac{1}{3}}\right\} .
$$

Unfortunately $q$ is not a norm, since it does not satisfy the triangle inequality. Using the energy estimate in [11], and repeating the calculations in Lemma 2.5 we only get $q(u+v) \leq e^{2 / 3}(q(u)+q(v))$.

\section{On the Topology of $\delta \mathscr{F}$}

Theorem 3.1. $(\delta \mathscr{F},\|\cdot\|)$ is a Banach space.

Proof. Lemmata 2.4 and 2.7 , and Corollary 2.6 shows that $(\delta \mathscr{F},\|\cdot\|)$ is a normed vector space. It remains to show completeness.

Suppose $\left(u_{n}\right)$ is a Cauchy sequence in $\delta \mathscr{F}$. For each integer $k$ there is an integer $n_{k}$ such that $\left\|u_{n}-u_{m}\right\|<2^{-k}$ for $n, m>n_{k}$. We choose the $n_{k}$ 's such that $n_{k+1}>n_{k}$.

We have $u_{n_{k}}=u_{n_{1}}+\left(u_{n_{2}}-u_{n_{1}}\right)+\cdots+\left(u_{n_{k}}-u_{n_{(k-1)}}\right)$. Since $u_{n_{j}} \in \delta \mathscr{F}$ for $j=1, \ldots, k$ we can write $u_{n_{j}}-u_{n_{j-1}}=\phi_{j}^{1}-\phi_{j}^{2}$, for $\phi_{j}^{1}, \phi_{j}^{2} \in \mathscr{F}$, where 
the $\phi_{j}^{1}$ and $\phi_{j}^{2}$ are chosen such that

$$
\left\|u_{n_{j}}-u_{n_{j-1}}\right\|=\inf \left(\int\left(d d^{c}\left(\varphi^{1}+\varphi^{2}\right)\right)^{n}\right)^{1 / n} \geq\left(\int\left(d d^{c}\left(\phi_{j}^{1}+\phi_{j}^{2}\right)\right)^{n}\right)^{1 / n}-2^{-j-1} .
$$

Then we have

$$
\begin{aligned}
u_{n_{k}} & =u_{n_{1}}+\left(\phi_{2}^{1}-\phi_{2}^{2}\right)+\cdots+\left(\phi_{k}^{1}-\phi_{k}^{2}\right) \\
& =u_{n_{1}}+\left(\phi_{2}^{1}+\cdots+\phi_{k}^{1}\right)-\left(\phi_{2}^{2}+\cdots+\phi_{k}^{2}\right)
\end{aligned}
$$

and since $\sum_{j=2}^{k} \phi_{j}^{1} \in \mathscr{P} \mathscr{S} \mathscr{H}^{-}(\Omega)$ is a decreasing sequence and

$$
\begin{aligned}
& \left(\int\left(d d^{c}\left(\sum_{j=2}^{k} \phi_{j}^{1}\right)\right)^{n}\right)^{1 / n} \\
& \quad \leq\left(\int\left(d d^{c}\left(\sum_{j=2}^{k} \phi_{j}^{1}+\phi_{j}^{2}\right)\right)^{n}\right)^{1 / n} \leq \sum_{j=2}^{k}\left(\int\left(d d^{c}\left(\phi_{j}^{1}+\phi_{j}^{2}\right)\right)^{n}\right)^{1 / n} \\
& \quad \leq \sum_{j=2}^{k}\left(\left\|u_{n_{j}}-u_{n_{j-1}}\right\|+2^{-j-1}\right)^{1 / n}=\sum_{j=2}^{k}\left(2^{-j}+2^{-j-1}\right)^{1 / n}<\frac{1}{\sqrt[n]{2}-1} .
\end{aligned}
$$

Thus $\sum_{j=2}^{k} \phi_{j}^{1}$ is an decreasing sequence of plurisubharmonic functions with bounded total mass, and in the same way $\sum_{j=2}^{k} \phi_{j}^{2}$ is. Therefore $u_{n_{k}}$ is convergent to some $u \in \delta \mathscr{F}$, and since $\left(u_{n}\right)$ is a Cauchy sequence $u_{n} \rightarrow u$.

Lemma 3.2. $\mathscr{F}$ is closed in the topology of $\delta \mathscr{F}$.

Proof. Take any Cauchy-sequence $\left(u_{m}\right)$ in $\mathscr{F}$. Choose a suitable sparse subsequence $\left(u_{m}^{\prime}\right)$, then $u_{p}=u_{0}+u_{1}-u_{0}+\cdots+u_{p}-u_{p-1}$, and by the exact same reasoning as in the proof of completeness for $\delta \mathscr{F}$, we get that $u_{p} \rightarrow u \in \mathscr{F}$.

Proposition 3.3. The continuous functions are not dense in $\delta \mathscr{F}$. Furthermore $\delta \mathscr{F}$ is not separable.

Proof. Let us denote the Lelong number of $u$ at $x$ with $v(u, x)$. The Lelong number at the origin is of course a linear functional on all of $\delta \mathscr{F}$, furthermore $v(\cdot, 0)$ is a continuos linear functional on $\delta \mathscr{F}$, by Theorem 4.3 or directly by the estimate:

$$
(2 \pi v(u, x))^{n} \leq\left(d d^{c} u\right)^{n}(\{x\}),
$$

for functions $u \in \mathscr{F}$ (see e.g. [10]). 
For all functions $u \in \mathscr{P} \mathscr{S} \mathscr{H} \cap \mathscr{C}$ we have $v(u, 0)=0$, thus $\log |z|$ can not be approximated by continuos functions in our topology.

For the second statement of the proposition, let us assume that $\delta \mathscr{F}$ is indeed separable. Let $\left\{u_{i}\right\}$ be a dense subset of $\delta \mathscr{F}$. It is well known that the set where the Lelong number is positive for a given function $u$, is of Lebesgue measure zero. Thus the union of the sets where the Lelong number is positive for functions from $\left\{u_{i}\right\}$ is also of Lebesgue-measure zero. Take any point $x$ not in this union, i.e. $v\left(u_{i}, x\right)=0$ for all $u_{i}$ 's, and then we see that $v(z)=\int_{\Omega} \log |z| \delta_{x}$ cannot be approximated from functions in $\left\{u_{i}\right\}$.

A vector space $L$ over $\mathrm{R}$ with an order structure defined by a binary relation " $\leq$ " being reflexive, transitive and anti-symmetric is called an ordered vector space over $\mathrm{R}$ if the relation satisfies:

(1) translation-invariance, $x \leq y \Longrightarrow x+z \leq y+z$ for all $x, y, z \in L$

(2) $x \leq y \Longrightarrow \lambda x \leq \lambda y$ for all $x, y \in L$ and $\lambda>0$.

Clearly every vector space of real-valued functions $f$ on a parameter set $X$ is an ordered vector space under the natural order $f \leq g$ if $f(x) \leq g(x)$ for all $x \in X$.

If $L$ is a topological vector space, and an ordered vector space, we say that if is an ordered topological vector space if the positive cone $C=\{x \mid x \geq 0\}$ is closed on $L$. In particular $\delta \mathscr{F}$ is an ordered topological vector space since $\{u \in \delta \mathscr{F} \mid u \geq 0\}$ is closed on the topology of $\delta \mathscr{F}$.

A comprehensive treatise of ordered topological vector spaces is found in the book of Schaefer and Wolff [18].

It is natural to ask wether $\delta \mathscr{F}$ has even more ordered structure.

Remember that a vector lattice is an ordered vector space $L$ over $\mathrm{R}$ such that $\sup (x, y)$ and $\inf (x, y)$ exist for every pair $(x, y) \in L \times L$. For a vector lattice $L$ set $|x|=\sup (x,-x)$. Of course $\delta \mathscr{F}$ is a vector lattice $\operatorname{since} \sup (u, v)=$ $\max (u, v)$ exist and the same for infimum.

Given a topological vector space $L$ over R, with a vector lattice structure, a set $X \subset L$ is called solid if $x \in X$ and $|x| \leq|y|$ imply that $y \in X$.

We call $L$ locally solid if it has a 0 -neighbourhood base of solid sets, i.e. the norm is compatible with the lattice structure.

Unfortunately $\delta \mathscr{F}$ is not locally solid. It suffices to show that the the unit ball B $\subset L$ is not solid, (see e.g. [18] or [17]), and this is showed in the example below.

EXAmple 3.4. Consider the function $f(\zeta)=\max (\log |\zeta|,-1)-$ $\max (\log |\zeta|,-1 / 2)$ in the unit-disc $D$ in $C^{1}$. We have $\|\log |\zeta|\|=\pi$, and $|f| \leq|\log | \zeta||$. 
Since $\max (\log |\zeta|,-1)=p_{\mu}$, and $\max (\log |\zeta|,-1 / 2)=p_{\nu}$, where $\mu$ and $v$ are the Lebesgue measure on the circles $\left\{|\zeta|=e^{-1}\right\}$ and $\left\{|\zeta|=e^{-1 / 2}\right\}$, and therefore have disjunct support we can calculate that $\|f\|=\pi+\pi$. Thus $\delta \mathscr{F}(\mathrm{D})$ is not locally solid. In particular: $\delta \mathscr{F}$ is not a so-called Banach lattice. (A Banach lattice is a locally solid Banach space.)

\section{The dual space}

Let us denote the topological dual of $\delta \mathscr{F}$ by $(\delta \mathscr{F})^{\prime}$.

It is natural to ask which elements of the dual can be given by Borel measures.

Theorem 4.1. Take $\psi \in \mathscr{F}$. Suppose $\Psi \in(\delta \mathscr{F})^{\prime}$ is given by

$$
\Psi(u)=\int d d^{c} u \wedge\left(d d^{c} \psi\right)^{n-1},
$$

then $\|\Psi\|=\|\psi\|^{n-1}$, and if $\psi \neq 0$ there is no Borel measure on $\Omega$ such that $\Psi(u)=\int u d \mu$.

Proof. Let $u \in \mathscr{F}$. According to Lemma 2.2 we have

$$
\Psi(u)=\int d d^{c} u \wedge\left(d d^{c} \psi\right)^{n-1} \leq\left(\int\left(d d^{c} u\right)^{n}\right)^{\frac{1}{n}}\left(\int\left(d d^{c} \psi\right)^{n}\right)^{\frac{n-1}{n}} .
$$

Thus $\Psi(u) \leq\|u\| \cdot\|\psi\|^{n-1}$. Take $f \in \delta \mathscr{F}$ and choose any $u, v \in \mathscr{F}$ such that $f=u-v$, then

$$
\begin{aligned}
|\Psi(f)| & =|\Psi(u-v)| \leq|\Psi(u)|+|\Psi(v)|=\Psi(u)+\Psi(v) \\
& =\Psi(u+v) \leq\left(\int\left(d d^{c}(u+v)\right)^{n}\right)^{\frac{1}{n}} \cdot\|\psi\|^{n-1},
\end{aligned}
$$

and we get that

$$
|\Psi(f)| \leq \inf _{u-v=f ; u, v \in \mathscr{F}}\left(\int\left(d d^{c}(u+v)\right)^{n}\right)^{1 / n} \cdot\|\psi\|^{n-1}=\|f\| \cdot\|\psi\|^{n-1}
$$

On the other hand, take $u=\|\psi\|^{-1} \psi$. Then $\|u\|=1$ and

$$
\Psi(u)=\int d d^{c}\left(\|\psi\|^{-1} \psi\right) \wedge\left(d d^{c} \psi\right)^{n-1}=\|\psi\|^{n-1} .
$$

Thus

$$
\|\Psi\|=\sup _{\|f\|=1}|\Psi(f)|=\|\psi\|^{n-1}
$$


To see that $\Psi$ is not given by a Borel measure, take $u, v \in \mathscr{F}$ such that $u=v$ near $\partial \Omega$. Then

$$
\int_{\Omega} d d^{c} u \wedge\left(d d^{c} \psi\right)^{n-1}=\int_{\Omega} d d^{c} v \wedge\left(d d^{c} \psi\right)^{n-1},
$$

by "Stokes' theorem", and if $\Psi(u)=\int_{\Omega} u d \mu$ then $\int_{\Omega}(v-u) d \mu=0$. Since $\mathscr{C}_{0}^{\infty} \subset \delta \mathscr{E}_{0}$ (see Lemma 3.1, [10]) it follows that $d \mu$ has its support on the boundary of $\Omega$. But then $\Psi(u)=0$ for all $u \in \mathscr{E}_{0}$. Take a sequence $\left\{u_{j}\right\} \subset \mathscr{E}_{0}$ such that $u_{j} \searrow \psi$, and by continuity we get $\int_{\Omega}\left(d d^{c} \psi\right)^{n}=0$, thus $\psi=0$.

ExAmple 4.2. Suppose $q>1$. Let $g \in L^{q}(\Omega)$. For any $u \in \mathscr{F}(\Omega)$, define $T(u)=\int u g d V$, then $T \in(\delta \mathscr{F})^{\prime}$.

Proof. From [12] we have for every $u \in \mathscr{F}$ with $\int\left(d d^{c} u\right)^{n} \leq 1$ there is a constant $A$, depending only on $\Omega$ such that $\int e^{-u} d V \leq A$. Thus $u \in L^{p}, \forall p$.

THEOREM 4.3. If $T$ is a linear functional on $\delta \mathscr{F}$ such that $T(x) \geq 0$, for all $x \in \mathscr{F}$, then $T$ is continuous.

Proof. Take a bounded sequence $\left\{f_{k}\right\} \subset \delta \mathscr{F}$, such that $\left\|f_{k}\right\|<M$. By construction there is $x_{k}, y_{k} \in \mathscr{F}$ such that $f_{k}=x_{k}-y_{k}$, and $\left\|x_{k}+y_{k}\right\|<M+1$. We have $\left\|x_{k}\right\|=\left\|f_{k}+y_{k}\right\| \leq\left\|f_{k}\right\|+\left\|y_{k}\right\| \leq M+\left\|y_{k}\right\| \leq M+\left\|x_{k}+y_{k}\right\| \leq$ $2 M+1$, where the second to last inequality follows from that $y_{k} \geq x_{k}+y_{k}$, thus $\int\left(d d^{c} y_{k}\right)^{n} \leq \int\left(d d^{c}\left(x_{k}+y_{k}\right)\right)^{n}$,

If $T$ is bounded on all bounded sequences $\left\{x_{k}\right\} \subset \mathscr{F}$ then $\left|T\left(f_{k}\right)\right|=$ $\left|T\left(x_{k}\right)-T\left(y_{k}\right)\right| \leq\left|T\left(x_{k}\right)\right|+\left|T\left(y_{k}\right)\right|$, and $T\left(f_{k}\right)$ would be bounded as well.

Suppose $T$ is not continuous. Then there has to be a bounded sequence $\left\{f_{k}\right\} \subset \delta \mathscr{F}$ such that $\left\{T\left(f_{k}\right)\right\}$ is not bounded. Thus there has to be a bounded sequence $\left\{x_{k}\right\}$ in $\mathscr{F}$ such that $T\left(x_{k}\right)>k>0$.

Now define $\phi=\sum_{k=1}^{\infty} k^{-2} x_{k}$. Since $\mathscr{F}$ is a convex cone and $\left\{x_{k}\right\}$ is bounded $\phi \in \mathscr{F}$. Note that $T(\phi)=T\left(\sum_{1}^{p} x_{k}\right)+T\left(\sum_{p+1}^{\infty} x_{k}\right) \geq T\left(\sum_{1}^{p} x_{k}\right)$, since $T \geq 0$ on $\mathscr{F}$. But then $T(\phi) \geq \sum_{1}^{p} k^{-1}$, for all positive numbers $p$, i.e. $T=+\infty$, and we have a contradiction.

Let us recall the notion of dual cones.

Definition 4.4. If $C$ is a cone in the topological vector space $L$, the dual cone $C^{\prime}$ of $C$ is defined to be the set

$$
C^{\prime}=\{T \in L \mid T(u) \geq 0 \text { if } u \in C\} .
$$

THEOREM 4.5. $(\delta \mathscr{F})^{\prime}=\mathscr{F}^{\prime}-\mathscr{F}^{\prime}=\delta \mathscr{F}^{\prime}$.

Proof. This follows more or less immediately from [16], (see also Lemma 1 p. $218[18]$ ), since one can show that $\mathscr{F}$ is a so called normal cone, but to avoid 
giving the rather abstract definitions of normal cones, we give a self contained proof.

Take $T \in(\delta \mathscr{F})^{\prime}$ and define $p: \mathscr{F} \rightarrow \mathbf{R}_{+}$by $p(u):=\sup \{T(v) \mid u \leq v \leq$ $0\}$. By the linearity of $T, p(\lambda u)=\lambda p(u)$, for $\lambda \geq 0$, and since $\{\phi \mid u+v \leq$ $\phi \leq 0\} \supset\{\phi \mid u \leq \phi \leq 0\}+\{\phi \mid v \leq \phi \leq 0\}, p(u+v) \geq p(u)+p(v)$ also. Thus the set $V=\{(t, u) \mid 0 \leq t \leq p(u)\} \subset \mathbf{R} \times \delta \mathscr{F}$ is a convex cone.

Clearly $\mathbf{R} \times \delta F$ is a normable space. Take a sequence $\left\{u_{k}\right\} \subset \mathscr{F}$ such that $\left\|u_{k}\right\| \rightarrow 0$, as $k \rightarrow \infty$. If $\varphi \in \mathscr{F}$ and $u_{k} \leq \varphi \leq 0$ then $\int\left(d d^{c} \varphi\right)^{n} \leq \int\left(d d^{c} u_{k}\right)^{n}$, and hence $\|\varphi\| \leq\left\|u_{k}\right\|$, thus $p\left(u_{k}\right) \rightarrow 0$, as $k \rightarrow \infty$ by the continuity of $T$. We conclude that $(1,0) \notin \bar{V}$.

Since $\delta \mathscr{F}$ is locally convex there is a closed real hyperplane $H=\{t, u) \mid$ $h(t, x)=-1\}$, separating $\bar{V}$ and $(1,0)$ where we can choose $h$ such that $h \geq 0$ on $V$ and $h(1,0)=-1$. Since $(\mathbf{R} \times \delta \mathscr{F})^{\prime}$ is algebraically isomorphic with $(\mathrm{R} \oplus \delta \mathscr{F})^{\prime}$, (see Theorem 4.3 p. 137, [18]) we have $h(t, u)=\alpha t+g(u)$. Now $h(1,0)=\alpha=-1$.

Since $(0, u) \in V$, for all $u \in \mathscr{F}$, and $g \in(\delta \mathscr{F})^{\prime}$, we have $g(u) \geq 0$ on $\mathscr{F}$ according to our choice of $H$. $V$ was chosen such that $(p(u), u) \in V$, hence $h(p(u), u)=-p(u)+g(u) \geq 0$, and we get $T(u) \leq p(u) \leq g(u)$. To sum up: $T=g-(g-T)$, where $g-T \geq 0$. Note that by Theorem 4.3, linear operators that are positive on $\mathscr{F}$ are continuous.

We can extend the definition of the Monge-Ampère operator to the whole of $\delta \mathscr{F}$. Suppose $u \in \delta \mathscr{F}$, then $u=u_{1}-u_{2}$, for some $u_{1}, u_{2} \in \mathscr{F}$, and we can define $\left(d d^{c} u\right)^{n}=\sum_{j=0}^{n}(-1)^{j}\left(\begin{array}{l}n \\ j\end{array}\right)\left(d d^{c} u_{1}\right)^{n-j} \wedge\left(d d^{c} u_{2}\right)^{j}$. To see that this definition is independent of the choice of the functions from $\mathscr{F}$, suppose $u=u_{1}-u_{2}=v_{1}-v_{2}$, and that $h \in \mathscr{E}_{0}$. Then

$$
\begin{array}{rl}
\int h & d d^{c}\left(u_{1}-u_{2}\right) \wedge \cdots \wedge d d^{c}\left(u_{1}-u_{2}\right) \\
& =\int\left(u_{1}-u_{2}\right) d d^{c} h \wedge d d^{c}\left(u_{1}-u_{2}\right) \wedge \cdots \wedge d d^{c}\left(u_{1}-u_{2}\right) \\
& =\int\left(v_{1}-v_{2}\right) d d^{c} h \wedge d d^{c}\left(u_{1}-u_{2}\right) \wedge \cdots \wedge d d^{c}\left(u_{1}-u_{2}\right) \\
& =\int h d d^{c}\left(v_{1}-v_{2}\right) \wedge d d^{c}\left(u_{1}-u_{2}\right) \wedge \cdots \wedge d d^{c}\left(u_{1}-u_{2}\right),
\end{array}
$$

and continuing iteratively we have

$$
\int h d d^{c}\left(u_{1}-u_{2}\right) \wedge \cdots \wedge d d^{c}\left(u_{1}-u_{2}\right)=\int h d d^{c}\left(v_{1}-v_{2}\right) \wedge \cdots \wedge d d^{c}\left(v_{1}-v_{2}\right) .
$$


COROLlARY 4.6. The following functionals are all continuous on $\delta \mathscr{F}$ :

- The total mass of the Monge-Ampère measure.

- Demailly's generalized Lelong numbers $v\left(d d^{c} u, \varphi\right)$ for the current $d d^{c} u$ with weight $\varphi$.

For a definition of $v(T, \varphi)$ — the Lelong number of the current $T$ with weight $\varphi$ see [14].

\section{Comparison with delta-subharmonic functions}

Let us turn our attention to the class of delta-subharmonic functions in domains in $\mathrm{C}^{n}$.

If we have a generating family of seminorms on a Fréchet space $X$ and if $K$ is a closed convex cone in $X$ we can turn $K$ into a Fréchet space with topology defined by the seminorms

$$
\|f\|_{j}=\inf \left\{|g|_{j}+|h|_{j} ; f=g-h, \text { for } g \text { and } h \text { in } K\right\}, \quad j \in \mathbf{N},
$$

where $|\cdot|_{j}$ are a generating family of seminorms on $X$.

DEFINITION 5.1. The set $\delta m$. Let $m(\Omega)$ be the set of positive measures that can be written as $\mu=\Delta \varphi$, for some $\varphi \in \mathscr{P} \mathscr{S} \mathscr{H}(\Omega)$. We denote the space of differences from this cone by $\delta m(\Omega)$ as usual.

Since any open domain $\Omega \subset C^{n}$ is para-compact it suffices to define a seminorm for any compact $K \Subset \Omega$ and generate the topology from these seminorms.

Using the topology on $\delta \mathscr{P} \mathscr{S} \mathscr{H}$, the delta-plurisubharmonic functions, defined in the introduction we have a continuity property of the Laplace operator.

TheOREM 5.2. Assume that $\Omega$ is pseudoconvex then $\delta m(\Omega)$ is a Fréchet space with seminorms defined by

$$
\|\mu\|_{K}=\inf \left(\int_{K} \mu_{1}+\mu_{2} \mid \mu=\mu_{1}-\mu_{2}, \mu_{1}, \mu_{2} \in m(\Omega)\right), \quad K \Subset \Omega .
$$

Furthermore the Laplace operator $\Delta: \delta \mathscr{P} \mathscr{S} \mathscr{H}(\Omega) \mapsto \delta m(\Omega)$ is continuos.

Proof. Cf. [7]. (By assuming that $\Omega$ is pseudoconvex we don't have to deal with some homotopy intricacies.)

Definition 5.3. The set $\delta M$. Let us denote the set of all positive real Borel measures on $\Omega$ by $M(\Omega)$, and the signed real Borel measures as $\delta M(\Omega)$. Then the total variation of a measure $\mu \in \delta M(\Omega)$ is by Jordan's decomposition theorem given as

$$
|\mu|=\inf \left(\int_{\Omega} \mu_{1}+\mu_{2} \mid \mu=\mu_{1}-\mu_{2}, \mu_{1}, \mu_{2} \in M(\Omega)\right) .
$$


We will view $\delta M(\Omega)$ as a Banach space with norm defined by the equation above.

Let $\Delta$ denote the Laplacian as a map from $\delta \mathscr{F}$ to $\delta M$. Clearly $\Delta$ is a linear map. Continuity of the map, however, turns out to be more subtle.

Theorem 5.4. Suppose $\Omega$ is a strict pseudoconvex domain with $\mathscr{C}^{\infty}{ }_{\text {-smooth }}$ boundary, then the map $\Delta: \delta \mathscr{F} \rightarrow \delta M$ is continuous. lem:

Proof. According to [6] the solution $\varphi \in \mathscr{P} \mathscr{S} \mathscr{H}(\Omega)$ to the Dirichlet prob-

$$
\begin{cases}\left(d d^{c} \varphi\right)^{n}=1 & \text { on } \Omega \\ \varphi=-\|z\|^{2} & \text { on } \partial \Omega\end{cases}
$$

satisfy $\varphi \in \mathscr{C}^{\infty}(\bar{\Omega})$. Thus it follows that $\|z\|^{2}+\varphi \in \mathscr{E}_{0}(\Omega)$.

Direct calculation gives that

$$
d d^{c} u \wedge\left(d d^{c}\|z\|^{2}\right)^{n-1}=4^{n-1}(n-1) ! \Delta u .
$$

Thus we have that

$$
\begin{aligned}
4^{n-1}(n-1) ! \int_{\Omega} \Delta u & =\int_{\Omega} d d^{c} u \wedge\left(d d^{c}\|z\|^{2}\right)^{n-1} \\
& \leq \int_{\Omega} d d^{c} u \wedge\left(d d^{c}\left(\|z\|^{2}+\varphi\right)\right)^{n-1} \\
& \leq\left(\int_{\Omega}\left(d d^{c} u\right)^{n}\right)^{1 / n}\left(\int_{\Omega}\left(d d^{c}\left(\|z\|^{2}+\varphi\right)\right)^{n}\right)^{(n-1) / n} \\
& \leq C \cdot\left(\int_{\Omega}\left(d d^{c} u\right)^{n}\right)^{1 / n}
\end{aligned}
$$

for some positive constant $C$. The second inequality above follows from Lemma 2.2.

Take $u \in \delta \mathscr{F}$ and any $\epsilon>0$, then there is a choice of $u_{1}, u_{2}$ such that $u=u_{1}-u_{2}$ where $\int\left(d d^{c}\left(u_{1}+u_{2}\right)\right)^{n}<\|u\|^{n}+\epsilon$. According to the calculation above we have

$$
\int_{\Omega} \Delta u_{1}+\Delta u_{2}=\int_{\Omega} \Delta\left(u_{1}+u_{2}\right) \leq C^{\prime} \cdot\left(\int_{\Omega}\left(d d^{c}\left(u_{1}+u_{2}\right)\right)^{n}\right)^{1 / n}<C^{\prime}\|u\|+\epsilon
$$

for some constant $C^{\prime}$, not depending on $\epsilon$. Let $\epsilon \rightarrow 0$ and the theorem follows.

Unfortunately, continuity of $\Delta$ does not hold in general, in particular not where the boundary of the domain is "flat", as can be seen from the following example. 
ExAmPle 5.5. Let $u_{k}=\max \left(k \log \left|z_{1}\right|,(1 / k) \log \left|z_{2}\right|\right)$. Then there is a constant $c$, not depending on $k$, such that

$$
\int_{\mathrm{D}^{2}} \Delta u_{k} \geq c \cdot k
$$

but

$$
\int_{D^{2}}\left(d d^{c}\left(u_{k}\right)\right)^{2}=(2 \pi)^{-2} .
$$

Proof. Take $\chi_{1}, \chi_{2} \in \mathscr{C}_{0}^{\infty}(\mathrm{D})$, where $\mathrm{D}$ is the unit disc. Then

$$
\begin{aligned}
\int_{\mathrm{D}^{2}} \chi_{1} \chi_{2} \Delta u_{k} & =\int_{\mathrm{D}^{2}} u_{k}\left(z_{1}, z_{2}\right) \Delta\left(\chi_{1}\left(z_{1}\right) \chi_{2}\left(z_{2}\right)\right) \\
& =\int_{\mathrm{D}^{2}} u_{k}\left(z_{1}, z_{2}\right)\left(\chi_{2}\left(z_{2}\right) \Delta_{1} \chi_{1}\left(z_{1}\right)+\chi_{1}\left(z_{1}\right) \Delta_{2} \chi_{2}\left(z_{2}\right)\right) \\
& =\int_{\mathrm{D}} \chi_{2} \int_{\mathrm{D}} u_{k} \Delta_{1} \chi_{1}+\int_{\mathrm{D}} \chi_{1} \int_{\mathrm{D}} u_{k} \Delta_{2} \chi_{2} \geq \int_{\mathrm{D}} \chi_{2} \int_{\mathrm{D}} u_{k} \Delta_{1} \chi_{1} \\
& =\int_{\mathrm{D}} \chi_{2} \int_{\mathrm{D}} \chi_{1} \Delta_{1} u_{k} .
\end{aligned}
$$

Take $\chi_{2}$ such that $\chi_{2} \equiv 1$ on $\mathrm{D}(1 / 2)$. For $z_{2}$ fixed with $\left|z_{2}\right|<1 / 2$ we know that $\Delta_{1} \max \left(k \log \left|z_{1}\right|, k^{-1} \log \left|z_{2}\right|\right)$ is $k$ times the (normalized) Lebesgue measure on the circle $\left\{z_{1} \in \mathrm{C} ;\left|z_{1}\right|^{k^{2}}=\left|z_{2}\right|\right\}$. Choose $\chi_{1}$, depending on $k$, such that $\chi_{1} \equiv 1$ at least where $\left|z_{1}\right| \leq\left(\frac{1}{2}\right)^{1 / k^{2}}$. After making all these choices we have

$$
\int_{\mathrm{D}} \chi_{2} \int_{\mathrm{D}} \chi_{1} \Delta_{1} u_{k}=\int_{\mathrm{D}} \chi_{2} \frac{k}{2 \pi} d z_{2} \wedge d \bar{z}_{2}>c \cdot k
$$

for some constant $c$, independent of $k$.

It is well known that $\int_{\mathrm{D}^{2}}\left(d d^{c}\left(u_{k}\right)\right)^{2}=(2 \pi)^{-2}$.

REMARK 5.6. Let $\Omega$ be a hyperconvex domain and take a sequence $\left\{u_{k}\right\}$ in $\mathscr{F}(\Omega)$ such that $\int \Delta u_{k}$ diverges. Exhaust $\Omega$ with smooth, strict pseudoconvex domains from inside, then Theorem 5.4 implies that the Laplace mass of the $u_{k}$ has to be pushed out towards the boundary.

Let $U(0, f)$ be the Perron-Bremermann function of $f$, i.e. the largest locally bounded plurisubharmonic function that has boundary values at most $f$. (See e.g. [9])

In his Doctoral Thesis, Åhag [1] generalized the notion $\mathscr{F}_{p}(f)$ of energy classes with "boundary data" $f$, from [9], and introduced $\mathscr{F}(f, \Omega)$. Assume that

$$
\lim _{\Omega \ni z \rightarrow \zeta} U(0, f)=f(\zeta)
$$


for every $\zeta \in \partial \Omega$, then we define the $\mathscr{F}(f, \Omega)$ to be set of plurisubharmonic functions on $\Omega$ such that there is a $\varphi \in \mathscr{F}$ such that $U(0, f) \geq u \geq \varphi+$ $U(0, f)$.

EXAMPLE 5.7. Let

$$
u(z)=\sum_{k=1}^{\infty} \max \left(\log \left|z_{1}\right|, k^{-4} \log \left|z_{2}\right|\right) .
$$

Then $u \in \mathscr{F}\left(\mathrm{D}^{2}\right)$, but $\int_{\mathrm{D}^{2}} \Delta u=+\infty$. Furthermore take $f=\left|z_{2}\right|^{2}-1$, then $f \in \mathscr{C}^{\infty}\left(\overline{\mathrm{D}}^{2}\right)$ and $\left(d d^{c} f\right)^{2}=0$ but $\left(d d^{c}(u+f)\right)^{2}$ is not bounded on $\mathrm{D}^{2}$.

Proof. Let $u_{k}=\max \left(\log \left|z_{1}\right|, k^{-4} \log \left|z_{2}\right|\right)$, then $\int_{\mathrm{D}^{2}}\left(d d^{c}\left(u_{k}\right)\right)^{2}=$ $\left(2 \pi k^{2}\right)^{-2}$.

By Lemma 2.5 we get

$$
\int_{D^{2}}\left(d d^{c}\left(\sum_{k=1}^{N} u_{k}\right)\right)^{2} \leq\left(\sum_{k=1}^{N}\left(\int\left(d d^{c} u_{k}\right)^{2}\right)^{1 / 2}\right)^{2}=\left(\sum_{k=1}^{N} \frac{1}{2 \pi k^{2}}\right)^{2} \leq \frac{\pi^{2}}{144},
$$

thus $u \in \mathscr{F}$, and $u+f \in \mathscr{F}(f)$. But we have

$$
\int d d^{c} u_{k} \wedge d d^{c}\left(\left|z_{2}\right|^{2}-1\right)=\int d d^{c} u_{k} \wedge\left(2 i d z_{2} \wedge d \bar{z}_{2}\right)=16 \int \Delta_{1} u_{k}>c,
$$

where the constant $c$ is independent of $k$, by the inequality in Example 5.5 above. Thus

$$
\int\left(d d^{c}\left(f+\sum_{k=1}^{N} u_{k}\right)\right)^{2} \geq 2 \int \sum_{k=1}^{N} d d^{c} u_{k} \wedge d d^{c}\left(\left|z_{2}\right|^{2}-1\right) \geq N,
$$

and we get that the total mass of $u+f$ diverges.

To ensure that if $u \in \mathscr{F}(f)$ we have $\int_{\Omega}\left(d d^{c} u\right)^{n}<+\infty$ Åhag introduced the concept of compliant functions $f$. A continuous function $f: \partial \Omega \rightarrow \mathbf{R}$ is said to be compliant if the Perron-Bremermann function $U(0, f)$ satisfies $U(0, f)=f$ on the boundary and $\int\left(d d^{c}(U(0, f)+U(0,-f))\right)^{n}<+\infty$.

Åhag proved, using the smoothness result for the Monge-Ampère operator of Caffarelli-Kohn-Nirenberg-Spruck [6], that under the assumption that $\Omega$ is strict pseudoconvex and smooth, any smooth boundary function is compliant.

In relation to this Åhag [2] has posed the following problem:

Problem. Suppose $\Omega$ is hyperconvex, $f \in \mathscr{C}^{\infty}$, and $f=U(0, f)$. If $u \in \mathscr{F}(\Omega)$, is $\int_{\Omega}\left(d d^{c}(u+f)\right)^{n}<+\infty$ ? 
According to Example 5.7 above the answer to this problem is no, not always. To see this it simply suffices to take $f$ and $u$ as in the example.

Since the dual of the space $\delta M$ is well understood it would be nice to pull back $(\delta M)^{\prime}$ to $(\delta F)^{\prime}$. At the moment this does not seems feasible considering the example below.

Example 5.8. Let $B$ be the unit ball in $C^{2}$. The inverse Laplace-operator $\Delta^{-1}: \delta M(\mathrm{~B}) \mapsto \delta \mathscr{F}(\mathrm{B})$ is not continuous. Let $u\left(z_{1}, z_{2}\right)=-\left(1-\left|z_{1}\right|^{2}\right)^{1 / 2}+$ $\left|z_{2}\right|$. Then $u \in \mathscr{P} \mathscr{S} \mathscr{H} \cap \mathscr{C}(\mathrm{B})$, and $u=0$ on the boundary of the ball. Away from the $z_{1}$-axis we have that

$$
4 \partial \bar{\partial} u=\left(\frac{\left|z_{1}\right|^{2}}{\left(1-\left|z_{1}\right|^{2}\right)^{3 / 2}}+\frac{2}{\left(1-\left|z_{1}\right|^{2}\right)^{1 / 2}}\right) d z_{1} \wedge d \bar{z}_{1}+\frac{1}{\left|z_{2}\right|} d z_{2} \wedge d \bar{z}_{2} .
$$

Thus setting $r=\left|z_{1}\right|$ and $\rho=\left|z_{2}\right|$ we calculate

$$
\begin{aligned}
\int_{\mathrm{B}} \Delta u d V & =4(2 \pi)^{2} \int_{0}^{1} \int_{0}^{\sqrt{1-r^{2}}}\left(\frac{r^{2}}{\left(1-r^{2}\right)^{3 / 2}}+\frac{2}{\left(1-r^{2}\right)^{1 / 2}}+\frac{1}{\rho}\right) r \rho d \rho d r \\
& =4 \pi^{2} .
\end{aligned}
$$

In [5] Błocki pointed out that even though $\left(d d^{c}\left(-\left(1-\left|z_{1}\right|^{2}\right)^{1 / 2}\right)\right)^{2}=\left(d d^{c}\left|z_{2}\right|\right)^{2}$ $=0$ we still have that $\int_{\mathrm{B}}\left(d d^{c} u\right)^{n} d V=+\infty$, since for any real number $0<$ $a<1$, we have

$$
\begin{aligned}
\int_{\mathrm{B}}\left(d d^{c} u\right)^{n} d V \geq \frac{1}{16}(2 \pi)^{2} & \int_{0}^{a} \int_{0}^{\sqrt{1-r^{2}}} \frac{2-r^{2}}{\left(1-r^{2}\right)^{3 / 2}} r d \rho d r \\
& =\frac{\pi^{2}}{4} \int_{0}^{a} \frac{2 r-r^{3}}{1-r^{2}} d r=\frac{\pi^{2}}{8}\left(a^{2}-\log \left(1-a^{2}\right)\right)
\end{aligned}
$$

which of course diverges as $a$ tends to one.

\section{REFERENCES}

1. Åhag, Per, The Complex Monge-Ampère Operator on Bounded Hyperconvex Domains, $\mathrm{PhD}$ thesis, Umeå University, 2002.

2. Åhag, Per, Personal communication, 2003.

3. Arsove, Maynard G., Functions representable as differences of subharmonic functions, Trans. Amer. Math. Soc. 75 (1953), 327-365.

4. Błocki, Zbigniew, Estimates for the complex Monge-Ampère operator, Bull. Polish Acad. Sci. Math. 41(2) (1994), 151-157.

5. Błocki, Zbigniew, Personal communication, 1997.

6. Caffarelli, L., Kohn, J. J., Nirenberg, L., and Spruck, J., The Dirichlet problem for nonlinear second-order elliptic equations II, Complex Monge-Ampère, and uniformly elliptic, equations, Comm. Pure Appl. Math. 38(2) (1985), 209-252. 
7. Cegrell, Urban, Delta-plurisubharmonic functions, Math. Scand. 43(2) (1979), 343-352, 1978.

8. Cegrell, Urban, On the space of delta-convex functions and its dual, Bull. Math. Soc. Sci. Math. R. S. Roumanie (N.S.) 22(70)(2) (1978), 133-139.

9. Cegrell, Urban, Pluricomplex energy, Acta Math. 180(2) (1998), 187-217.

10. Cegrell, Urban, The general definition of the complex Monge-Ampère operator, Ann. Inst. Fourier (Grenoble) 54 (2004).

11. Cegrell, Urban, and Persson, Leif, An energy estimate for the complex Monge-Ampère operator, Ann. Polon. Math. 67(1) (1997), 95-102.

12. Cegrell, Urban, and Zeriahi, Ahmed, Subextension of plurisubharmonic functions with bounded Monge-Ampère mass, C. R. Math. Acad. Sci. Paris 336(4) (2003), 305-308.

13. Cepedello Boiso, Manuel, Two characterizations of super-reflexive Banach spaces by the behaviour of differences of convex functions, J. Funct. Anal. 191(1) (2002), 1-16.

14. Demailly, Jean-Pierre, Nombres de Lelong généralisés, théorèmes d'intégralité et d'analyticité, Acta Math. 159(3-4) (1987), 153-169.

15. Kiselman, Christer O., Fonctions delta-convexes, delta-sousharmoniques et delta-plurisousharmoniques, In Séminaire Pierre Lelong (Analyse), année 1975/76, pages 93-107, Lecture Notes in Math. 578 (1977).

16. Krein, M., Propriétés fondamentales des ensembles coniques normaux dans l'espace de Banach, C. R. (Doklady) Acad. Sci. URSS (N.S.) 28 (1940), 13-17.

17. Meyer-Nieberg, Peter, Banach Lattices, Universitext, 1991.

18. Schaefer, H. H., and Wolff, M. P., Topological vector spaces, Graduate Texts in Math. 3, second edition, 1999.

19. Wiklund, Jonas, Topics in Pluripotential Theory, PhD thesis, Umeå University, 2004.

MATEMATISKA INSTITUTIONEN

UMEÅ UNIVERSITET

S-901 87 UME̊

SWEDEN

E-mail: urban.cegrell@math.umu.se,jonas.wiklund@math.umu.se 